\title{
THE CONJUGATE OF A SINGULAR FUNCTIONAL DIFFERENTIAL OPERATOR
}

\author{
L. M. HALL \\ Department of Mathematics \& Statistics \\ University of Nebraska \\ Lincoln, Nebraska 68588
}

(Received November 11, 1977)

ABSTRACT. An explicit representation of the conjugate of a singular functional differential operator is given. Some theorems and their corollaries are proven.

\section{INTRODUCTION.}

In (1) the author, together with L. J. Grimm, studied the solvability of complex functional differential systems of the form

$$
A(z) y^{\prime}(z)+B(z) y(z)+C(z) y(\lambda z)+E(z) y^{\prime}(\lambda z)=g(z),
$$

in the unit disk. The following Banach spaces provided the framework for their study. Let $D$ denote the open unit disk and $C$ the unit circle in the complex plane, and define $A_{p}$ to be the Banach space of functions $v(z)$ analytic in $D$ and p times continuously differentiable on DUC, with norm

$$
\|v(z)\|_{p}=\left\{\max \left|v^{(i)}(z)\right|, 0 \leq i \leq p, z \in C\right\}
$$


Define $A_{p, n}$ to be the Banach space of $n$-vector functions $y(z)=\left(y^{1}(z), y^{2}(z), \ldots, y^{n}(z)\right)^{T}$ where $y^{k}(z) \varepsilon A_{p}, 1 \leq k \leq n$, with norm

$$
\|\left. y(z)\right|_{p} ^{n}=\left\{\max || y^{k}(z) \|_{p}, 1 \leq k \leq n\right\} .
$$

Now let $L: A_{1, n} \rightarrow A_{0, n}$ be given by

$$
(L y)(z)=A(z) y^{\prime}(z)+B(z) y(z)+C(z) y(\lambda z)+E(z) y^{\prime}(\lambda z),
$$

where $A, B, C$, and $E$ are $n \times n$ matrices of functions in $A_{0}$ and $\lambda$ is a complex constant, $0<|\lambda|<1$. Hence for $g \in A_{0, n}$ equation (1.1) can be written as $L y=g$. In order to apply the principal theorem of (1) the analytic solutions of the conjugate system $L^{\star} f=0$ must be found. These solutions were calculated for certain special cases in (1) and (2) and in (3) the author has proved that, in the scalar case, every analytic solution of $L^{*} f=0$ belongs to the Hardy space $\mathrm{H}^{2}$. These results were obtained by showing that $f$ is an analytic solution of $L * f=0$ if and only if, for real $r, \lim _{r \rightarrow 1-} B(L y, f ; r)=0$ for all y $\varepsilon A_{1, n}$, and then solving the latter equation or system for $f . \quad\left(B(g, h ; z) \equiv \sum_{k=0}^{\infty} g_{k} \cdot h_{k} z^{k}\right.$, where $g(z)$ and $h(z)$ are $n$-vector functions analytic in D with power series representations $\sum_{k=0}^{\infty} g_{k} z^{k}$ and $\sum_{k=0}^{\infty} h_{k} z^{k}$, respectively. $B(g, h ; z)$ is sometimes called the Hadamard product of $\mathrm{g}$ and $\mathrm{h}$ and can also be represented in integral form (see (5)). In this paper an explicit representation of the operator $L^{*}$ will be given.

In (1.2) let $A(z)=z^{D}, D$ a constant diagonal matrix with nonnegative integer elements, and also let $E(z) \equiv 0$. If $f(z)$ is an $n$-vector function analytic at $z=0$ with series representation $\sum_{j=0}^{\infty} f_{j} z^{j}$, define the k-truncation of $f, k$ an integer, to be

$$
\left(T_{k} f\right)(z)=\sum_{j=0}^{k-1} f_{j} z^{j}
$$


In case $k$ is a negative integer or zero, define $\left(T_{k} f\right)(z) \equiv 0$. Let $Q$ be an $n \times n$ diagonal matrix with integer elements, $Q=\operatorname{diag}\left(q_{1}, \ldots, q_{n}\right)$, and denote the vector components of $f(z)$ by superscripts, 1.e., $f(z)=\left(f^{1}(z), f^{2}(z), \ldots, f^{n}(z)\right)^{T}$. Then define the Q-truncation of $f$ to be

$$
\left(T Q_{Q} f\right)(z)=\left(\left(T_{q_{1}} f^{l}\right)(z), \ldots,\left(T_{q_{n}} f^{n}\right)(z)\right)^{T},
$$

Note that $\left(T_{k} f\right)(z)=\left(T_{k I} f\right)(z)$. Finally, if $G(z)$ is an $n \times n$ matrix of functions analytic at $z=0$, define $\hat{G}(z)=G^{T}(z)-(D-I) z^{D-I}$, where $D$ is as described above. The following theorem give the representation of $L *$.

\section{MAIN RESULTS.}

THEOREM 1. An n-vector function $f(z)$, analytic at $z=0$, is a solution of $L * f=0$ if and only if $f(z)$ is a solution of

$$
\begin{aligned}
& z^{2 I-D}\left[f(z)-\left(T_{D-I} f\right)(z)\right]^{\prime}+\hat{B}\left(\frac{1}{z}\right) f(z)+C^{T}\left(\frac{1}{\lambda z}\right) f(\lambda z) \\
& -\sum_{k=1}^{\infty}\left[\hat{B}_{k}\left(T_{k} f\right)(z)\right] z^{-k}-\sum_{k=1}^{\infty}\left[C_{k}^{T}\left(T_{k} f\right)(\lambda z)\right](\lambda z)^{-k}=0 .
\end{aligned}
$$

PROOF. It is sufficient to show that analytic solutions of both $L^{\star} f=0$ and (2.1) satisfy the same recursion formulas. Since $f$ is an analytic solution of $L * f=0$ if and only if $f$ satisfies $\lim _{r \rightarrow 1^{-}} B(L y, f ; r)=0$ for all y $\varepsilon A_{1, n}$, the Hadamard product can be written in series form to yield one recursion formula, and equation (2.1) yields the other when the series $j_{j=0}^{\infty} f_{j} z^{j}$ is substituted for $f(z)$. Equivalence is then obtained by straightforward vector-matrix algebra.

The next theorem, which can be proved in the same way as Theorem 1, guarantees that, for the operator $\mathrm{L}$, the second conjugate operator can be replaced by the operator itself in Theorem 3.1 of (1).

THEOREM 2. The function $y(z) \in A_{1, n}$ is a solution of $L^{* *} y=0$ if and only if 
$y(z)$ is a solution of $L y=0$.

Since every function in $A_{1, n}$ also belongs to $A_{0, n}^{*}$, the operators $L$ and $L^{*}$ will act on the same set of functions if the domain of $L^{*}$ is restricted to those members of $A_{0, n}^{*}$ which are also members of $A_{1, n}$. Deonte $L^{*}$ restricted in this way by $L_{R}^{*}$. The following result is a corollary to Theorem 1 .

COROLLARY 1. Let $B(z)$ and $C(z)$ be constant symmetric matrices and let $D=I$. Then $L_{R}^{*}=L$ in the sense that a function is a solution of $L_{R}^{*} f=0$ if and only if it is also a solution of Ly $=0$.

The next three corollaries of Theorem 1 deal with the type of singularity possessed by $L y=0$ and $L^{*} f=0$ when $B(z)$ and $C(z)$ are constant matrices and $D=\operatorname{diag}\left\{d_{1}, d_{2}, \ldots, d_{n}\right\}$ with $d_{i}=0,1$, or 2 for $i=1, \ldots, n$. In this case the equations $L y=0$ and $L^{*} f=0$ have the forms

$$
\begin{aligned}
& z^{D} y^{\prime}(z)+B y(z)+C y(\lambda z)=0, \\
& z^{2 I-D} f^{\prime}(z)+B^{T} f(z)+C^{T} f(\lambda z)=0,
\end{aligned}
$$

respectively. The term simple singularity will be used as defined in Hartman (4), p. 73 .

COROLLARY 2. Equation (2.2) has a simple singularity at $z=0$ if and only if equation (2.3) has a simple singularity at $z=0$.

COROLLARY 3. $z=0$ is an ordinary point for equation (2.3) if and only if $\mathrm{D}=2 \mathrm{I}$.

COROLLARY 4. If $z=0$ is an ordinary point for equation (2.2) then $z=0$ is a nonsimple singularity for equation (2.3).

Let $L^{*}$ be expressed as $L^{*}=\tilde{L}+M$, where $\tilde{L}$ and $M$ are defined as follows:

$$
\begin{aligned}
& (\tilde{L} f)(z)=z^{2 I-D} f^{\prime}(z)+B\left(\frac{1}{z}\right) f(z)+C^{T}\left(\frac{1}{\lambda z}\right) f(\lambda z) \text {, } \\
& (M f)(z)=-\left(T_{D}-I^{f}\right)^{\prime}(z)-\sum_{k=1}^{\infty} \hat{B}_{k}\left(T_{k} f\right)(z) z^{-k}-\sum_{k=1}^{\infty} C_{k}^{T}\left(T_{k} f\right)(\lambda z)(\lambda z)^{-k} .
\end{aligned}
$$


Note that in case $B(z)$ and $C(z)$ are constant matrices and $d_{i}=0,1$, or 2 then $\tilde{L}=L^{*}$ and $M=0$. The following result is another corollary of Theorem 1 .

COROLLARY 5. If either $\mathrm{B}(\mathrm{z})$ or $\mathrm{C}(\mathrm{z})$ has at least one non-polynomial element, then $z=0$ is an irregular singular point for the equation $\tilde{L} f=0$.

PROOF. If the hypothesis of the corollary is satisfied, then either $\hat{B}\left(\frac{1}{z}\right)$ or $C^{T}\left(\frac{1}{\lambda z}\right)$ has an essential singularity at $z=0$. Hence $z=0$ is an irregular singular point for $\tilde{\mathrm{L}} \mathrm{f}=0$.

ACKNOWLEDGMENT. This research was partially supported by NSF Grant MPS75-06368.

\section{REFERENCES}

1. Grimm, L. J. and L. M. Hall. An Alternative Theorem for Singular Differential Systems, J. Diff. Equations 18 (1975) 411-422.

2. Grimm, L. J. and L. M. Hall. Solvability of Nonhomogeneous Singular Differential Equations, Mathematica Balkanica, to appear.

3. Hal1, L. M. A Characterization of the Cokernel of a Singular Fredholm Differential Operator, J. Diff. Equations 24 (1977) 1-7.

4. Hartman, Philip. Ordinary Differential Equations, The Johns Hopkins University, Ba1timore, Maryland, 1973.

5. Taylor, A. E. Banach Spaces of Functions Analytic in the Unit Circle, I, II, Studia Math. 11 (1950) 145-170; Studia Math. 12 (1951) 25-50.

KEY WORDS AND PHRASES. Singular differential operators, complex functional differential systems, representation theorems. 


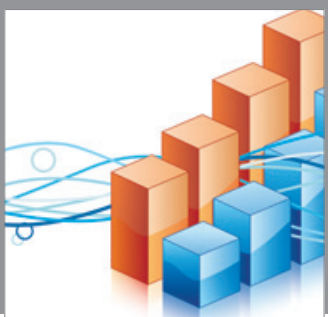

Advances in

Operations Research

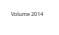

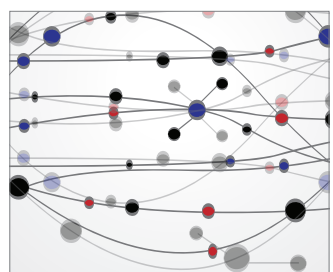

\section{The Scientific} World Journal
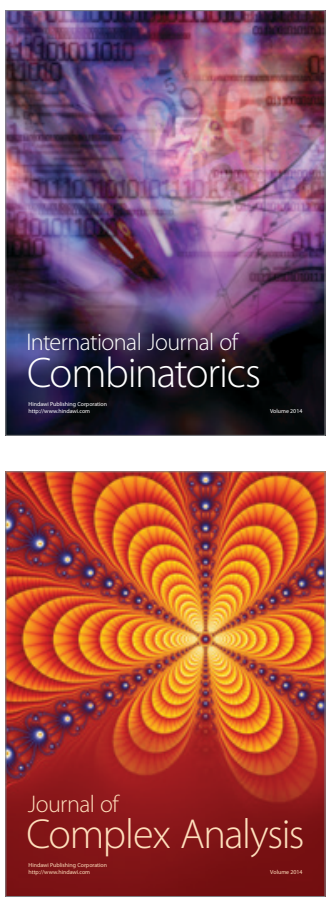

International Journal of

Mathematics and

Mathematical

Sciences
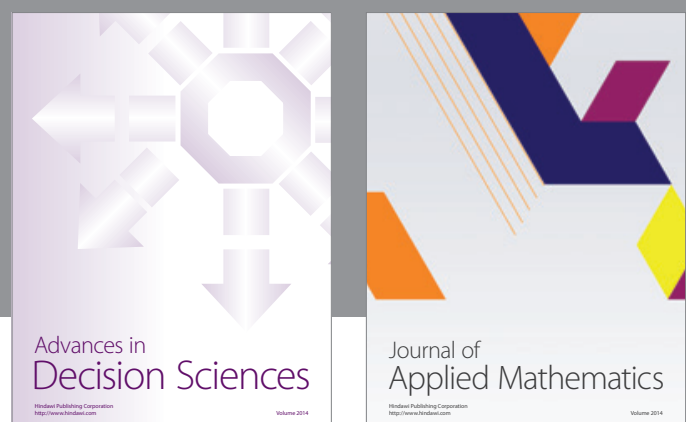

Journal of

Applied Mathematics
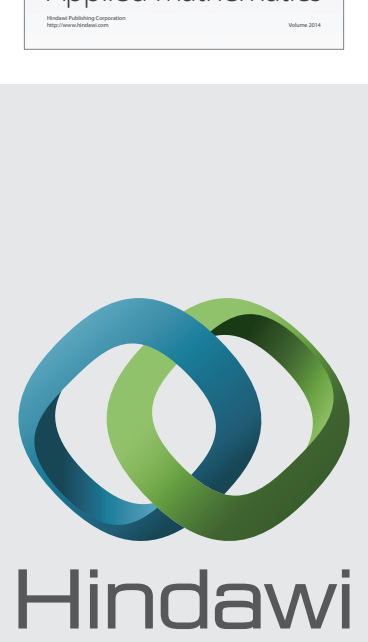

Submit your manuscripts at http://www.hindawi.com
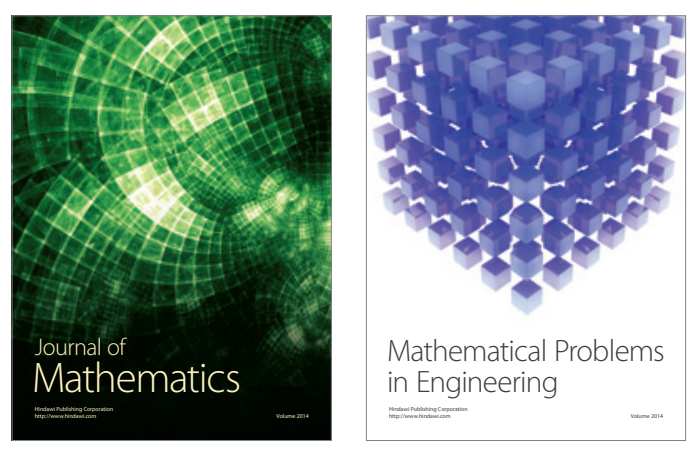

Mathematical Problems in Engineering
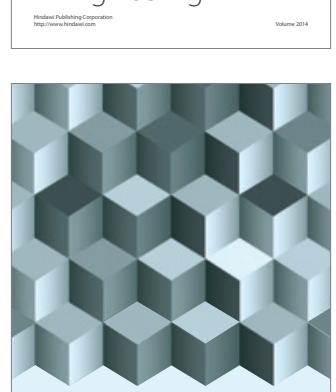

Journal of

Function Spaces
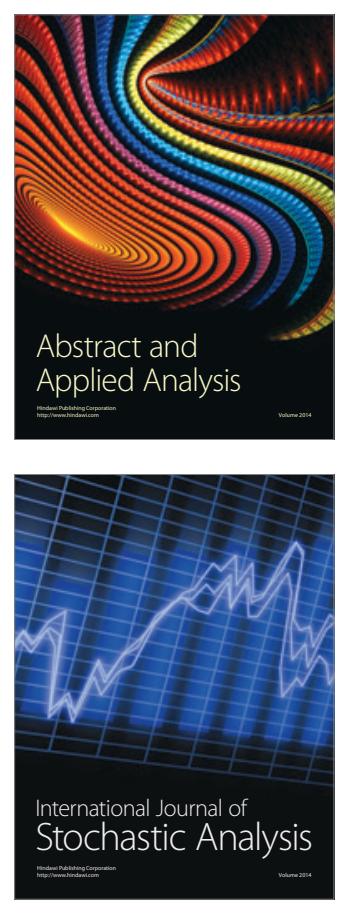

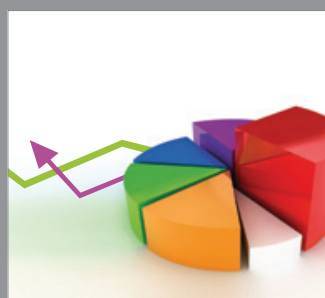

ournal of

Probability and Statistics

Promensencen
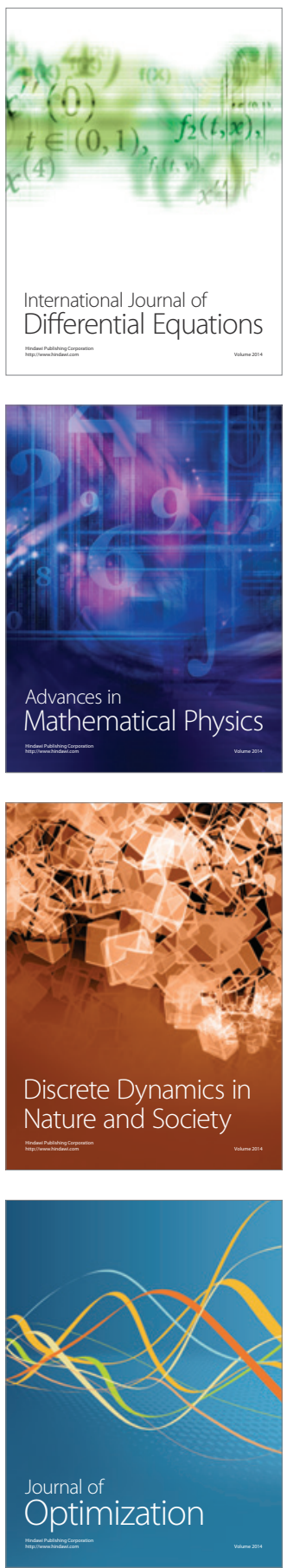